\title{
Exponential Riesz bases, discrepancy of irrational rotations and BMO
}

\author{
Gady Kozma and Nir Lev
}

\begin{abstract}
We study the basis property of systems of exponentials with frequencies belonging to 'simple quasicrystals'. We show that a diophantine condition is necessary and sufficient for such a system to be a Riesz basis in $L^{2}$ on a finite union of intervals. For the proof we extend to BMO a theorem of Kesten about the discrepancy of irrational rotations of the circle.
\end{abstract}

\section{Introduction}

1.1. Sampling and interpolation. A band-limited signal is an entire function $F$ of exponential type, square-integrable on the real axis. According to the classical PaleyWiener theorem, $F$ is the Fourier transform of an $L^{2}$-function supported by a bounded (measurable) set $S \subset \mathbb{R}$, which is called the spectrum of $F$. We shall denote by $P W_{S}$ the Paley-Wiener space of all functions $F \in L^{2}(\mathbb{R})$ which are Fourier transforms of functions from $L^{2}(S)$,

$$
F(t)=\int_{S} f(x) e^{-2 \pi i t x} d x, \quad f \in L^{2}(S) .
$$

A discrete set $\Lambda \subset \mathbb{R}$ is called a set of sampling for $P W_{S}$ if every signal with spectrum in $S$ can be reconstructed in a stable way from its 'samples' $\{F(\lambda), \lambda \in \Lambda\}$, that is, there are positive constants $A, B$ such that the inequalities

$$
A\|F\|_{L^{2}(\mathbb{R})} \leqslant\left(\sum_{\lambda \in \Lambda}|F(\lambda)|^{2}\right)^{1 / 2} \leqslant B\|F\|_{L^{2}(\mathbb{R})}
$$

hold for every $F \in P W_{S}$. Also, $\Lambda$ is called a set of interpolation for $P W_{S}$ if every data $\left\{c_{\lambda}\right\} \in \ell^{2}(\Lambda)$ can be "transmitted" as samples, which means that there exists at least one function $F \in P W_{S}$ such that $F(\lambda)=c_{\lambda}(\lambda \in \Lambda)$.

The sampling and interpolation properties can also be formulated in terms of the exponential system

$$
E(\Lambda)=\{\exp 2 \pi i \lambda t, \lambda \in \Lambda\} .
$$

The sampling property of $\Lambda$ means that $E(\Lambda)$ is a frame in the space $L^{2}(S)$, while the interpolation holds when $E(\Lambda)$ is a Riesz-Fischer system in this space. It follows that $\Lambda$ is a set of both sampling and interpolation if and only if $E(\Lambda)$ forms a Riesz basis in $L^{2}(S)$. See $[\mathbf{3 1}]$ for a detailed discussion.

2000 Mathematics Subject Classification. 42C15, 11K38.

Key words and phrases. Riesz bases, Quasicrystals, Discrepancy, Bounded mean oscillation. 
Sampling and interpolation may also be discussed in the periodic setting. If $S$ is a measurable subset of the circle group $\mathbb{T}=\mathbb{R} / \mathbb{Z}$, then one can consider the frame or Riesz-Fischer properties in $L^{2}(S)$ of the exponential system $E(\Lambda)$, where $\Lambda \subset \mathbb{Z}$.

1.2. Density. The right inequality in (1) follows from the separation condition

$$
\inf _{\lambda, \lambda^{\prime} \in \Lambda, \lambda \neq \lambda^{\prime}}\left|\lambda-\lambda^{\prime}\right|>0
$$

which is also necessary for the interpolation, so this condition will always be assumed below. The lower and upper uniform densities of a separated set $\Lambda$ are defined respectively by

$$
\mathscr{D}^{-}(\Lambda)=\lim _{r \rightarrow \infty} \min _{a \in \mathbb{R}} \frac{\#(\Lambda \cap(a, a+r))}{r}, \quad \mathscr{D}^{+}(\Lambda)=\lim _{r \rightarrow \infty} \max _{a \in \mathbb{R}} \frac{\#(\Lambda \cap(a, a+r))}{r} .
$$

Landau obtained in [14] necessary conditions for sampling and interpolation in terms of these densities:

If $\Lambda$ is a set of sampling for $P W_{S}$, then $\mathscr{D}^{-}(\Lambda) \geqslant$ mes $S$.

If $\Lambda$ is a set of interpolation for $P W_{S}$, then $\mathscr{D}^{+}(\Lambda) \leqslant$ mes $S$.

Here $S$ is a bounded measurable set, and mes $S$ is the Lebesgue measure of $S$.

For "regularly" distributed sequences the two densities above coincide, and their common value, denoted $\mathscr{D}(\Lambda)$, is called the uniform density of $\Lambda$. It follows that:

If $E(\Lambda)$ is a Riesz basis in $L^{2}(S)$ then $\Lambda$ has a uniform density $\mathscr{D}(\Lambda)=\operatorname{mes} S$.

1.3. Universality problem. Olevskii and Ulanovskii posed in $[\mathbf{2 3}, \mathbf{2 4}]$ the following question: is it possible to find a "universal" set $\Lambda$ of given density, which provides a stable reconstruction of any signal whose spectrum has a sufficiently small Lebesgue measure? Similarly, does there exist $\Lambda$ of given density which is a set of interpolation in every $P W_{S}$ with mes $S$ sufficiently large?

It was proved in $[\mathbf{2 3}, \mathbf{2 4}]$ that no universal set of sampling or interpolation exists if the spectrum $S$ is allowed to be an arbitrary bounded measurable set. On the other hand it was also proved that under some topological restrictions on the spectra, universal sampling and interpolation does exist:

Given any $d>0$ there is a (separated) set $\Lambda \subset \mathbb{R}$ of density $\mathscr{D}(\Lambda)=d$, such that:

(i) $\Lambda$ is a set of sampling for $P W_{S}$ for every compact set $S \subset \mathbb{R}$, mes $S<d$.

(ii) $\Lambda$ is a set of interpolation for $P W_{S}$ for every open set $S \subset \mathbb{R}$, mes $S>d$.

In fact, this is a consequence of the following result:

Theorem A (Olevskii and Ulanovskii $[\mathbf{2 3}, \mathbf{2 4}])$. Given any $d>0$ one can find a discrete set $\Lambda \subset \mathbb{R}$ (a perturbation of an arithmetical progression) such that $E(\Lambda)$ is a Riesz basis in $L^{2}(S)$ for every set $S \subset \mathbb{R}$, mes $S=d$, which is the union of finitely many disjoint intervals such that the lengths of these intervals and the gaps between them are commensurable.

The latter result shows that one can construct a universal exponential Riesz basis $E(\Lambda), \Lambda \subset \mathbb{R}$, in the space $L^{2}(S)$ for a "dense" family of sets $S \subset \mathbb{R}$.

1.4. Simple quasicrystals. A different construction of universal sampling and interpolation sets, termed 'simple quasicrystals', was presented by Matei and Meyer in the papers $[\mathbf{1 8}, \mathbf{1 9}, \mathbf{2 0}]$. Their construction is based on the so-called 'cut and project' 
scheme introduced by Meyer in 1972 (see $[\mathbf{2 1}, \mathbf{2 2}]$ ). Here we shall restrict ourselves to the periodic setting, in which the simple quasicrystals take the following form.

Let $\alpha$ be an irrational real number, and consider the sequence of points $\{n \alpha\}, n \in \mathbb{Z}$, on the circle $\mathbb{T}=\mathbb{R} / \mathbb{Z}$. Given an interval $I=[a, b) \subset \mathbb{T}$ define the following subset of $\mathbb{Z}$,

$$
\Lambda(\alpha, I):=\{n \in \mathbb{Z}: a \leqslant n \alpha<b\} .
$$

It is well-known that the points $\{n \alpha\}$ are equidistributed on the circle $\mathbb{T}$. Moreover, they are well-distributed (see [13]). This implies that the set $\Lambda(\alpha, I)$ has a uniform density $\mathscr{D}(\Lambda(\alpha, I))=|I|$, where $|I|$ denotes the length of the interval $I$.

The set $\Lambda(\alpha, I)$ is a set of universal sampling and interpolation:

Theorem B (Matei and Meyer [19]).

(i) $E(\Lambda(\alpha, I))$ is a frame in $L^{2}(S)$ for every compact set $S \subset \mathbb{T}$, mes $S<|I|$.

(ii) $E(\Lambda(\alpha, I))$ is Riesz-Fischer in $L^{2}(S)$ for every open set $S \subset \mathbb{T}$, mes $S>|I|$.

A similar result in the non-periodic setting was formulated and proved in $[\mathbf{1 8}, \mathbf{2 0}]$. In the paper [19] the authors raised the question of what can be said in the "limiting case" when the measure of $S$ is equal to the density of $\Lambda$.

1.5. Riesz bases and quasicrystals. In the present paper we study the following problem. Is it true that the exponential system $E(\Lambda(\alpha, I))$ is a Riesz basis in $L^{2}(S)$ for certain subsets $S$ of the circle $\mathbb{T}$ ? Moreover, does it provide a universal Riesz basis for some "dense" family of sets?

Our first result shows that the question admits a positive answer provided that a diophantine condition, relating $\alpha$ and the length of the interval $I$, holds:

Theorem 1. Let $|I| \in \mathbb{Z} \alpha+\mathbb{Z}$. Then the exponential system $E(\Lambda(\alpha, I))$ is a Riesz basis in $L^{2}(S)$ for every set $S \subset \mathbb{T}$, mes $S=|I|$, which is the union of finitely many disjoint intervals whose lengths belong to $\mathbb{Z} \alpha+\mathbb{Z}$.

This result will be proved in a more general form in Section 3. It follows that if $|I| \epsilon$ $\mathbb{Z} \alpha+\mathbb{Z}$ then the exponential system $E(\Lambda(\alpha, I))$ is a universal Riesz basis for a "dense" family of sets, and, in particular, that properties (i) and (ii) in Theorem B can be strengthened as follows:

Corollary 1. Let $|I| \in \mathbb{Z} \alpha+\mathbb{Z}$. Suppose that $U \subset \mathbb{T}$ is an open set, $K$ is compact, $K \subset U$ and mes $K<|I|<\operatorname{mes} U$. Then one can find a set $S, K \subset S \subset U$, which is a finite union of intervals and such that $E(\Lambda(\alpha, I))$ is a Riesz basis in $L^{2}(S)$.

Theorem 1 also provides a new result about existence of exponential Riesz bases on finite unions of intervals. This problem was studied in $[\mathbf{2}, \mathbf{1 5}, \mathbf{1 6}, \mathbf{1 7}, \mathbf{3 0}]$ and in other papers, and it is still open in general. We discuss this in more details in Section 6.

Our second result complements the picture by clarifying the role of the diophantine assumption $|I| \in \mathbb{Z} \alpha+\mathbb{Z}$ in Theorem 1 . It turns out that this condition is not only sufficient, but also necessary, for the simultaneous sampling and interpolation property on "multiband spectra".

Theorem 2. Suppose that $|I| \notin \mathbb{Z} \alpha+\mathbb{Z}$. Then $E(\Lambda(\alpha, I))$ is not a Riesz basis in $L^{2}(S)$, for any set $S \subset \mathbb{T}$ which is the union of finitely many intervals. 
1.6. Discrepancy and Kesten's theorem. The proofs of the results above are based on the connection of the problem to the theory of equidistribution and discrepancy for the irrational rotation of the circle.

Let $\alpha$ be a fixed irrational number. Given an interval $I \subset \mathbb{T}$, we denote by $v(n, I)$ the number of integers $0 \leqslant k \leqslant n-1$ such that $k \alpha \in I$. The equidistribution of the points $\{n \alpha\}$ on the circle $\mathbb{T}$ means that

$$
\lim _{n \rightarrow \infty} \frac{v(n, I)}{n}=|I|,
$$

for every interval $I$. A quantitative measurement of this equidistribution is given by the discrepancy function, defined by

$$
D(n, I)=v(n, I)-n|I| .
$$

Thus we have $D(n, I)=o(n)$ as $n \rightarrow \infty$, and, in fact, it is not difficult to show that this estimate holds uniformly with respect to the interval $I$.

Better estimates for the discrepancy can be obtained based on diophantine properties of the number $\alpha$. For example, if $\alpha$ is a quadratic irrational then $D(n, I)=O(\log n)$, and this estimate is uniform with respect to $I$. On the other hand, it is known that for every $\alpha$ the lower bound

$$
\sup _{I \subset \mathbb{T}}|D(n, I)|>c \log n
$$

holds for infinitely many $n$ 's, where $c$ is a positive absolute constant (see [13]). Compare also to the case where $\alpha$ and $I$ are random $[\mathbf{1 0}, 11]$.

It was discovered, however, that for certain special intervals $I$ the discrepancy is bounded. Hecke proved in [6] that if $|I| \in \mathbb{Z} \alpha+\mathbb{Z}$ then $D(n, I)=O(1)$ as $n \rightarrow \infty$. It was conjectured by Erdös and Szüsz [3] that also the converse to Hecke's result should be true. This conjecture was confirmed by Kesten [12] who proved that if $D(n, I)=O(1)$

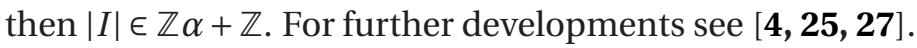

We will prove an extension of Kesten's theorem for bounded mean oscillation of the discrepancy. We say that a sequence of complex numbers $\left\{c_{n}\right\}$ has bounded mean oscillation, $\left\{c_{n}\right\} \in \mathrm{BMO}$, if for every $n<m$ one has

$$
\frac{1}{m-n} \sum_{k=n}^{m-1}\left|c_{k}-\frac{c_{n}+\cdots+c_{m-1}}{m-n}\right| \leqslant M
$$

for some constant $M$ independent of $n$ and $m$. Certainly, every bounded sequence belongs to BMO. On the other hand, it is well-known that BMO contains also unbounded sequences, for example $c_{n}=\log n$ is such a sequence.

We will prove the following generalization of Kesten's theorem.

Theorem 3. Let $\alpha$ be an irrational number, and $I \subset \mathbb{T}$ be an interval. If the sequence $\{D(n, I)\}, n=1,2,3, \ldots$, belongs to BMO, then $|I| \in \mathbb{Z} \alpha+\mathbb{Z}$.

This result along with Hecke's theorem reveals a dichotomy: either the discrepancy $D(n, I)$ is bounded, or it does not even have bounded mean oscillation. In Section 4 we will actually prove an extension of Theorem 3 for more general sets than single intervals (such as unions of several intervals), but, on the other hand, we cannot show that such a dichotomy still holds in this case. 
1.7. Meyer's duality. The link between the sampling and interpolation problems for the sets $\Lambda(\alpha, I)$ and the theory of discrepancy for irrational rotations is an idea due to Meyer, which we refer to as the 'duality principle'. Meyer's duality principle (see Section 2) enables us to reduce the problem about exponential Riesz bases in $L^{2}(S)$ to a similar problem in $L^{2}(I)$, where $I$ is a single interval. It is then possible to invoke known results about exponential Riesz bases in $L^{2}(I)$.

The proof of Theorem 1 (Section 3) consists of three main ingredients: Meyer's duality principle, a theorem of Avdonin [1] about exponential Riesz bases in $L^{2}(I)$ (an extension of Kadec's 1/4 theorem) and Hecke's result about discrepancy. In order to prove Theorem 2 (Section 5) we combine the duality principle with a theorem due to Pavlov [26], which describes completely the exponential Riesz bases in $L^{2}(I)$. Pavlov's theorem allows us to conclude that the discrepancy must be in BMO, and we can then apply an extension of Theorem 3 (Section 4).

1.8. Acknowledgements. We are grateful to A. M. Olevskii for introducing us to the concept of quasicrystals and for his suggestions which improved the presentation of this paper. We thank Itai Benjamini for referring us to Kesten's paper. We also thank Evgeny Abakumov, Jordi Marzo, Joaquim Ortega-Cerdà, Ron Peled, Omri Sarig, Mikhail Sodin and Armen Vagharshakyan for helpful comments and discussions.

\section{Meyer's duality principle}

2.1. Let $\alpha$ be a fixed irrational number. For any set $S \subset \mathbb{T}$ one can, in principle, consider the "Meyer set" based on $\alpha$ and $S$, defined by

$$
\Lambda(\alpha, S)=\{n \in \mathbb{Z}: n \alpha \in S\} .
$$

Meyer discovered a duality phenomenon connecting the sampling and interpolation properties of the sets $\Lambda(\alpha, S)$. This duality allowed to prove the results about sampling and interpolation such as Theorem B mentioned in the introduction. Meyer's duality principle is the starting point for our approach as well.

It will be convenient to introduce the following terminology.

Definition. A set $S \subset \mathbb{T}$ will be called a multiband set if it is the union of finitely many disjoint intervals. We will say that a multiband set $S$ is left semi-closed if each one of the intervals contains its left endpoint but not its right endpoint. Equivalently, $S$ is left semi-closed if the indicator function $\mathbb{1}_{S}$ is continuous from the right. We also define a right semi-closed multiband set, in a similar way. Finally, $S$ will be called semi-closed if it is either left semi-closed or right semi-closed.

We shall need a version of the duality principle which is suitable for our setting. Let $-\Lambda$ denote the set $\{-n: n \in \Lambda\}$. We will prove the following:

Lemma 2.1. Let $U$ be a semi-closed multiband set, and $V$ be a (not necessarily semiclosed) multiband set. Then:

(i) If $E(\Lambda(\alpha, V))$ is a frame in the space $L^{2}(U)$, then $E(-\Lambda(\alpha, U))$ is a Riesz-Fischer system in $L^{2}(V)$.

(ii) If $E(\Lambda(\alpha, V))$ is a Riesz-Fischer system in $L^{2}(U)$, then $E(-\Lambda(\alpha, U))$ is a frame in $L^{2}(V)$.

An immediate consequence of Lemma 2.1 is: 
Corollary 2.2. Let $U$ and $V$ be two multiband sets, where $U$ is semi-closed. If the exponential system $E(\Lambda(\alpha, V))$ is a Riesz basis in $L^{2}(U)$, then $E(-\Lambda(\alpha, U))$ is a Riesz basis in $L^{2}(V)$.

2.2. We choose and fix a function $\varphi(x)$ on $\mathbb{R}$, infinitely smooth, with compact support contained in the interval $(0,1)$, and such that $\int_{\mathbb{R}}|\varphi(x)|^{2} d x=1$. Let

$$
\widehat{\varphi}(\xi)=\int \varphi(x) e^{-2 \pi i \xi x} d x, \quad \xi \in \mathbb{R},
$$

be the Fourier transform of $\varphi$, which is a smooth and rapidly decreasing function. For each $0<\varepsilon<1$ define a function $\varphi_{\varepsilon}$ on the circle $\mathbb{T}$ by

$$
\varphi_{\varepsilon}(t)=\frac{1}{\sqrt{\varepsilon}} \varphi(t / \varepsilon), \quad 0 \leqslant t<1 .
$$

It follows that $\varphi_{\varepsilon}$ is an infinitely smooth function on $\mathbb{T}$, supported by $(0, \varepsilon)$, such that $\int_{\mathbb{T}}\left|\varphi_{\varepsilon}(t)\right|^{2} d t=1$, and the Fourier coefficients of $\varphi_{\varepsilon}$ are given by

$$
\widehat{\varphi}_{\varepsilon}(n)=\sqrt{\varepsilon} \widehat{\varphi}(\varepsilon n), \quad n \in \mathbb{Z} .
$$

The following two lemmas are essentially due to Matei and Meyer $[\mathbf{1 8}, \mathbf{2 0}]$.

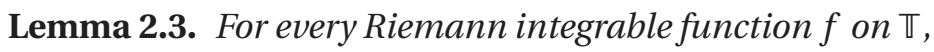

$$
\lim _{\varepsilon \rightarrow 0} \sum_{n \in \mathbb{Z}}\left|f(n \alpha) \widehat{\varphi}_{\varepsilon}(n)\right|^{2}=\int_{\mathbb{T}}|f(t)|^{2} d t .
$$

Lemma 2.4. Let $\left\{c_{n}\right\}$ be a sequence of complex numbers in $\ell^{1}(\mathbb{Z})$. Then

$$
\lim _{\varepsilon \rightarrow 0} \int_{S}\left|\sum_{n \in \mathbb{Z}} c_{n} \varphi_{\varepsilon}(t-n \alpha)\right|^{2} d t=\sum_{n \in \Lambda(\alpha, S)}\left|c_{n}\right|^{2},
$$

for every left semi-closed multiband set $S$.

Here, as always, $\alpha$ is an arbitrary irrational number. Lemma 2.3 is a consequence of the equidistribution of the points $\{n \alpha\}$ on $\mathbb{T}$. Lemma 2.4 is due to the fact that $\varphi_{\varepsilon}(t-n \alpha)$ is supported by a small right neighborhood of the point $n \alpha$. For the proof see [20]. Certainly, one can also get a version of Lemma 2.4 for right semi-closed multiband sets by choosing the function $\varphi$ supported by the interval $(-1,0)$ instead of $(0,1)$.

We will also need the following well-known fact:

Lemma 2.5 (See $[\mathbf{3 1}]$, p. 155). The exponential system $E(\Lambda)$ is Riesz-Fischer in $L^{2}(S)$ if and only if there is a positive constant $C$ such that the inequality

$$
\sum_{\lambda \in \Lambda}\left|c_{\lambda}\right|^{2} \leqslant C \int_{S}\left|\sum_{\lambda \in \Lambda} c_{\lambda} e^{2 \pi i \lambda t}\right|^{2} d t
$$

holds for every finite sequence of scalars $\left\{c_{\lambda}\right\}$.

2.3. Here we give the proof of Lemma 2.1. It is based on the proof from $[\mathbf{1 8 , 2 0}]$ but we find it useful to provide the reader with a self contained proof. Below we will assume that $U$ is a left semi-closed multiband set, but as we have remarked one can easily adapt the proof to the case when $U$ is right semi-closed.

Proof of Part (I) OF Lemma 2.1. Suppose that $E(\Lambda(\alpha, V))$ is a frame in the space $L^{2}(U)$. It is to be proved that $E(-\Lambda(\alpha, U))$ is a Riesz-Fischer system in $L^{2}(V)$. By Lemma 2.5 it will be enough to show that, if $f$ is a trigonometric polynomial

$$
f(t)=\sum_{n} c_{n} e^{-2 \pi i n t}
$$


such that $c_{n}=0$ unless $n \alpha \in U$, then

$$
\sum_{n}\left|c_{n}\right|^{2} \leqslant C \int_{V}|f(t)|^{2} d t
$$

Given such a trigonometric polynomial $f$ we define

$$
F_{\varepsilon}(t)=\sum_{n \in \mathbb{Z}} c_{n} \varphi_{\varepsilon}(t-n \alpha),
$$

where $\varphi$ is any function as in the previous section. Since $U$ is left semi-closed, it follows that $F_{\varepsilon}$ is supported by $U$ if $\varepsilon$ is sufficiently small. Since $E(\Lambda(\alpha, V))$ is a frame in $L^{2}(U)$, there is a constant $C$ such that

$$
\int_{U}\left|F_{\varepsilon}(t)\right|^{2} d t \leqslant C \sum_{n \in \Lambda(\alpha, V)}\left|\widehat{F}_{\varepsilon}(n)\right|^{2} .
$$

Again we take the limit as $\varepsilon \rightarrow 0$. Since $U$ is left semi-closed, Lemma 2.4 implies that the left hand side of (6) converges to $\sum\left|c_{n}\right|^{2}$. On the other hand, it is easy to see that $\widehat{F}_{\varepsilon}(n)=f(n \alpha) \widehat{\varphi}_{\varepsilon}(n)$. The right hand side of (6) can therefore be written as

$$
\sum_{n \in \mathbb{Z}}\left|f(n \alpha) \mathbb{1}_{V}(n \alpha) \widehat{\varphi}_{\varepsilon}(n)\right|^{2},
$$

and by Lemma 2.3 it converges to $\int_{V}|f(t)|^{2} d t$ as $\varepsilon \rightarrow 0$. This proves the first part of Lemma 2.1.

Proof of Part (II) of Lemma 2.1. Suppose that $E(\Lambda(\alpha, V))$ is a Riesz-Fischer system in $L^{2}(U)$. It is to be proved that $E(-\Lambda(\alpha, U))$ is a frame in $L^{2}(V)$, that is, we must show that the inequality

$$
\int_{V}|f(t)|^{2} d t \leqslant C \sum_{n \in \Lambda(\alpha, U)}|\widehat{f}(-n)|^{2}
$$

holds for every $f \in L^{2}(V)$. Since $V$ is a multiband set, it is actually enough to verify (7) for every infinitely smooth function $f$ supported by $V$. Given such $f$, define

$$
F_{\varepsilon}(t):=\sum_{n \in \mathbb{Z}} f(n \alpha) \widehat{\varphi}_{\varepsilon}(n) \exp 2 \pi i n t, \quad t \in \mathbb{T} .
$$

The fact that $f$ is supported by $V$ implies that only exponentials from $E(\Lambda(\alpha, V))$ have their coefficient non-zero in the series above. Since $E(\Lambda(\alpha, V))$ is a Riesz-Fischer system in $L^{2}(U)$, it follows from Lemma 2.5 that there is a constant $C$ such that

$$
\sum_{n \in \mathbb{Z}}\left|f(n \alpha) \widehat{\varphi}_{\varepsilon}(n)\right|^{2} \leqslant C \int_{U}\left|F_{\varepsilon}(t)\right|^{2} d t .
$$

Now we take the limit of (8) as $\varepsilon \rightarrow 0$. Lemma 2.3 implies that the left hand side of (8) tends to $\int|f(t)|^{2} d t$. On the other hand, substituting $f$ with its Fourier expansion in the definition of $F_{\varepsilon}$, it is easy to see that

$$
F_{\varepsilon}(t)=\sum_{n \in \mathbb{Z}} \widehat{f}(-n) \varphi_{\varepsilon}(t-n \alpha) .
$$

The coefficients $\{\widehat{f}(-n)\}$ belong to $\ell^{1}$, since $f$ is smooth. Since $U$ is left semi-closed we may use Lemma 2.4, which implies that the limit as $\varepsilon \rightarrow 0$ of the right hand side of (8) is equal to the right hand side of (7). This proves the second part of Lemma 2.1. 


\section{Exponential Riesz bases on multiband sets}

3.1. In this section we prove Theorem 1 . Let $I$ be an interval on $\mathbb{T}$ which is either left or right semi-closed. We will show that if the (necessary) diophantine condition $|I| \in \mathbb{Z} \alpha+\mathbb{Z}$ is satisfied, then the exponential system $E(\Lambda(\alpha, I))$ is a universal Riesz basis in $L^{2}(S)$ for a family of multiband sets $S$. In fact we will prove a somewhat more general result than formulated in the introduction.

Theorem 3.1. Suppose that $|I| \in \mathbb{Z} \alpha+\mathbb{Z}$. Then $E(\Lambda(\alpha, I))$ is a Riesz basis in $L^{2}(S)$ for every set $S \subset \mathbb{T}$, mes $S=|I|$, which satisfies the following condition: the indicator function $\mathbb{1}_{S}$ can be expressed as a finite linear combination of indicator functions of intervals $I_{1}, \ldots, I_{N}$ whose lengths belong to $\mathbb{Z} \alpha+\mathbb{Z}$, that is,

$$
\mathbb{1}_{S}(t)=\sum_{j=1}^{N} c_{j} \mathbb{1}_{I_{j}}(t), \quad c_{j} \in \mathbb{Z}, \quad\left|I_{j}\right| \in \mathbb{Z} \alpha+\mathbb{Z} \quad(1 \leqslant j \leqslant N) .
$$

Condition (9) is certainly satisfied if $S$ is the union of finitely many disjoint intervals with lengths in $\mathbb{Z} \alpha+\mathbb{Z}$. However, notice that other configurations are also possible. For example, consider the set $S$ of the form

$$
S=I_{1} \backslash I_{2}, \quad I_{2} \subset I_{1}, \quad\left|I_{1}\right|,\left|I_{2}\right| \in \mathbb{Z} \alpha+\mathbb{Z},
$$

which certainly satisfies the condition (9), but which is the union of two disjoint intervals whose lengths do not necessarily belong to $\mathbb{Z} \alpha+\mathbb{Z}$.

3.2. We may suppose, with no loss of generality, that $S$ is a left semi-closed multiband set, or, equivalently, that the intervals $I_{j}$ in the representation (9) are left semiclosed. The condition (9) plays its key role in the following lemma.

Lemma 3.2. Let $S$ be a left semi-closed multiband set satisfying (9). Then there is a bounded function $g: \mathbb{T} \rightarrow \mathbb{R}$, continuous from the right and with finitely many jump discontinuities, such that

$$
\mathbb{1}_{S}(t)-\operatorname{mes} S=g(t)-g(t+\alpha), \quad t \in \mathbb{T} .
$$

Proof. According to (9) the indicator function $\mathbb{1}_{S}$ is a linear combination of the functions $\mathbb{1}_{I_{j}}(1 \leqslant j \leqslant N)$. As equation (10) is linear as well, it will be enough to prove the lemma in the case when $S$ is each one of the intervals $I_{j}$. In other words, we may suppose that $S$ is a single interval whose length belongs to $\mathbb{Z} \alpha+\mathbb{Z}$. Moreover, by rotation, we may suppose that $S$ is an interval whose left endpoint is zero.

Let therefore $S=[0, n \alpha)$, where $n \in \mathbb{Z}$. For simplicity we shall suppose that $n$ is a positive integer, as the case when $n$ is negative is very similar. Let us denote by $\theta(t)$ the 1periodic function on $\mathbb{R}$, defined by $\theta(t)=t$ for $0 \leqslant t<1$. Considered as a function on the circle $\mathbb{T}$, this is a piecewise linear function, with slope +1 , and with a jump discontinuity of magnitude -1 at $t=0$. Set

$$
g(t):=\sum_{k=1}^{n} \theta(t-k \alpha), \quad t \in \mathbb{T},
$$

then $g$ is a bounded function, continuous from the right, and with finitely many jump discontinuities. We have

$$
g(t)-g(t+\alpha)=\theta(t-n \alpha)-\theta(t) .
$$


Observe that the function on the right hand side has the following properties: it has a jump of magnitude +1 at $t=0$ and another jump of magnitude -1 at $t=n \alpha$, it has derivative zero at all other points, and has zero integral on $\mathbb{T}$. These properties determine the function uniquely as $\mathbb{1}_{S}(t)-$ mes $S$, and so (10) is established.

Remark. If $S$ is a multiband set, then the condition (9) is not only sufficient, but also necessary, for the existence of a bounded measurable function $g(t)$ satisfying (10). This is a consequence of a result due to Oren [25] (see also [29]).

3.3. We turn to the proof of Theorem 3.1. The first step in the proof, based on Meyer's duality principle, is to reduce the problem about exponential Riesz bases in $L^{2}(S)$ to a similar problem in $L^{2}(I)$, where $I$ is a single interval. This allows us then to use a theorem of Avdonin [1] on exponential Riesz bases in $L^{2}(I)$. Below we formulate a special case of Avdonin's theorem, in a form which will be convenient in our setting.

Theorem 3.3 (Avdonin [1]). Let $I \subset \mathbb{R}$ be a bounded interval, and let

$$
\lambda_{j}=\frac{j+\delta_{j}+c}{|I|}, \quad j \in \mathbb{Z},
$$

where $c$ is a constant, and $\left\{\delta_{j}\right\}$ is a bounded sequence of real numbers. Suppose that there is a positive integer $N$ such that

$$
\sup _{n \in \mathbb{Z}}\left|\frac{1}{N} \sum_{j=1}^{N} \delta_{n+j}\right|<\frac{1}{4} .
$$

If the sequence $\Lambda=\left\{\lambda_{j}, j \in \mathbb{Z}\right\}$ is separated then $E(\Lambda)$ is a Riesz basis in $L^{2}(I)$.

Here the separation condition means that $\inf _{n \neq m}\left|\lambda_{n}-\lambda_{m}\right|>0$.

Note that the famous Kadec $1 / 4$ theorem (see [31]) corresponds to the special case when $N=1$ in Avdonin's theorem.

Proof of Theorem 3.1. Let $S$ be a multiband set satisfying the condition (9) and such that mes $S=|I|$. We must prove that $E(\Lambda(\alpha, I))$ is a Riesz basis in $L^{2}(S)$. Recall that $I$ is semi-closed by assumption. Hence by Corollary 2.2, with $U=-I$ and $V=S$, it will be enough to show that $E(\Lambda(\alpha, S))$ is a Riesz basis for $L^{2}(-I)$.

There is no loss of generality in assuming that $S$ is left semi-closed. Let us enumerate the set $\Lambda(\alpha, S)$ in an increasing order,

$$
\Lambda(\alpha, S)=\left\{\lambda_{j}, j \in \mathbb{Z}\right\}, \quad \cdots<\lambda_{-1}<0 \leqslant \lambda_{0}<\lambda_{1}<\lambda_{2}<\cdots,
$$

and take $g$ to be the function from Lemma 3.2. By the definition (3) of $\Lambda(\alpha, S)$ and according to (10), for any two integers $m<n$ we have

$$
\#(\Lambda(\alpha, S) \cap[m, n))=\sum_{k=m}^{n-1} \mathbb{1}_{S}(k \alpha)=(n-m) \operatorname{mes} S+g(m \alpha)-g(n \alpha) .
$$

Using this with $m=0, n=\lambda_{j}(j \geqslant 0)$ or with $m=\lambda_{j}, n=0(j<0)$ we get

$$
j=\lambda_{j} \operatorname{mes} S+g(0)-g\left(\lambda_{j} \alpha\right), \quad j \in \mathbb{Z} \text {. }
$$

Since mes $S=|I|$, this implies (11) with $\delta_{j}=g\left(\lambda_{j} \alpha\right)$ and $c=-g(0)$.

Observe that the perturbations $\left\{\delta_{j}\right\}$ are bounded, since $g$ is bounded. We may assume, by adding a constant to $g$ if necessary, that $\int_{S} g(t) d t=0$. Hence

$$
\lim _{m \rightarrow \infty} \frac{1}{m} \sum_{k=n+1}^{n+m} g(k \alpha) \mathbb{1}_{S}(k \alpha)=0,
$$


uniformly with respect to $n \in \mathbb{Z}$ (see [22], Chapter V, §6.3). It is then easy to see that (12) holds for a sufficiently large $N$. Moreover, the sequence $\Lambda(\alpha, S)$ is clearly separated, as the elements $\lambda_{j}$ are distinct integers. So the proof is concluded by Theorem 3.3.

Proof of Corollary 1. Let $|I| \in \mathbb{Z} \alpha+\mathbb{Z}$. First we claim that, being given any $\varepsilon>0$, one can find real numbers $\gamma_{1}, \ldots, \gamma_{s}$ in the segment $(0, \varepsilon)$, such that $\gamma_{j} \in \mathbb{Z} \alpha+\mathbb{Z}$ for each $j$, and $\sum \gamma_{j}=|I|$. Indeed, since $\mathbb{Z} \alpha+\mathbb{Z}$ is a dense subset of $\mathbb{R}$, it would be possible to choose numbers $\gamma_{1}, \ldots, \gamma_{s-1} \in(0, \varepsilon) \cap(\mathbb{Z} \alpha+\mathbb{Z})$ such that the difference

$$
|I|-\sum_{j=1}^{s-1} \gamma_{j}
$$

lies in $(0, \varepsilon)$, and then define $\gamma_{s}$ to be the value of (13), which is also in $\mathbb{Z} \alpha+\mathbb{Z}$.

Let now $U \subset \mathbb{T}$ be an open set, $K$ be compact, $K \subset U$ and mes $K<|I|<\operatorname{mes} U$. If $\varepsilon$ is sufficiently small then $K$ may be covered by disjoint intervals $I_{1}, \ldots, I_{s}$ contained in $U$, such that $\left|I_{j}\right|=\gamma_{j}$. Taking $S:=I_{1} \cup \cdots \cup I_{s}$ we have $K \subset S \subset U$, mes $S=|I|$, and $E(\Lambda(\alpha, I))$ is a Riesz basis in $L^{2}(S)$ by Theorem 1 .

\section{Ergodic sums, BMO and Kesten's theorem}

In this section we study the bounded mean oscillation of ergodic sums, first in an abstract setting, and then for the irrational rotation of the circle. In particular we will prove an extension of Theorem 3. The result obtained will then allow us to prove Theorem 2 in Section 5.

4.1. It will be convenient to start with an abstract setting. Let $H$ be a Hilbert space, and $U$ be a unitary operator on $H$. Given a vector $f \in H$ we consider its 'ergodic sums' defined by

$$
S_{n}:=f+U f+\cdots+U^{n-1} f .
$$

The von Neumann ergodic theorem asserts that the ratios $S_{n} / n$ converge to the projection of $f$ onto the closed subspace of $U$-invariant vectors. In particular, $f$ is perpendicular to this subspace if and only if $\left\|S_{n}\right\|=o(n)$ as $n \rightarrow \infty$.

A vector $f$ is called a coboundary if there exists $g \in H$ such that $f=g-U g$. In this case the ergodic sums have the form $S_{n}=g-U^{n} g$. This, of course, implies the boundedness of the ergodic sums, $\left\|S_{n}\right\|=O(1)$. However, it was proved by Robinson [28] that the latter condition is not only necessary but also sufficient for $f$ to be a coboundary. In fact, as the proof in $[\mathbf{2 8}]$ shows, the condition $(1 / N) \sum_{n=1}^{N}\left\|S_{n}\right\|^{2}=O(1)$ implies that $f$ is a coboundary.

We will prove the following

Theorem 4.1. For a vector $f$ to be a coboundary it is necessary and sufficient that the numbers $V_{N}$ (so-called 'variances') defined by

$$
V_{N}:=\frac{1}{N} \sum_{n=1}^{N}\left\|S_{n}-\frac{S_{1}+\cdots+S_{N}}{N}\right\|^{2}
$$

are bounded for $N=1,2,3, \ldots$. 
This result strengthens Robinson's theorem, as the assumption $V_{N}=O(1)$ is weaker than the condition $(1 / N) \sum_{n=1}^{N}\left\|S_{n}\right\|^{2}=O(1)$. This can be seen from the identity

$$
V_{N}=\frac{1}{N} \sum_{n=1}^{N}\left\|S_{n}\right\|^{2}-\left\|\frac{1}{N} \sum_{n=1}^{N} S_{n}\right\|^{2} .
$$

A key tool in [28] as well as in our proof of Theorem 4.1 is the spectral measure. Since the sequence $\left\{\left\langle U^{n} f, f\right\rangle\right\}$ is positive definite, by Herglotz's theorem (see [9]) there exists a positive, finite measure $\mu_{f}$ on the circle $\mathbb{T}$, such that

$$
\int_{\mathbb{T}} e^{2 \pi i n t} d \mu_{f}(t)=\left\langle U^{n} f, f\right\rangle, \quad n \in \mathbb{Z} .
$$

The measure $\mu_{f}$ is called the spectral measure of $f$ with respect to $U$. In the paper [28] the coboundaries were characterized also in terms of the spectral measure, in the following way: $f$ is a coboundary if and only if the integral

$$
\int_{\mathbb{T}} \frac{d \mu_{f}(t)}{\sin ^{2} \pi t}
$$

(with the integrand taking the value $+\infty$ at $t=0$ ) is finite. Theorem 4.1 is obtained by a combination of this result and the following

Lemma 4.2. For any $f \in H$ we have

$$
\lim _{N \rightarrow \infty} V_{N}=\frac{1}{4} \int_{\mathbb{T}} \frac{d \mu_{f}(t)}{\sin ^{2} \pi t},
$$

where the integral on the right hand side may be finite or infinite.

Proof. It follows from (15) that

$$
\|P(U) f\|^{2}=\int_{\mathbb{T}}\left|P\left(e^{2 \pi i t}\right)\right|^{2} d \mu_{f}(t)
$$

for every polynomial $P(z)=c_{0}+c_{1} z+\cdots+c_{n} z^{n}$. In particular, using (14) we can express the variance $V_{N}$ in terms of the spectral measure in the form

$$
V_{N}=\int_{\mathbb{T}} Q_{N}(t) d \mu_{f}(t),
$$

where $Q_{N}(t)$ is the trigonometric polynomial defined by

$$
Q_{N}(t):=\frac{1}{N} \sum_{n=1}^{N}\left|\sum_{k=0}^{n-1} e^{2 \pi i k t}-\frac{1}{N} \sum_{m=1}^{N} \sum_{k=0}^{m-1} e^{2 \pi i k t}\right|^{2} .
$$

By evaluation of the inner sums we get

$$
Q_{N}(t)=\frac{1}{N} \sum_{n=1}^{N}\left|\frac{1-e^{2 \pi i n t}}{1-e^{2 \pi i t}}-\frac{1}{N} \sum_{m=1}^{N} \frac{1-e^{2 \pi i m t}}{1-e^{2 \pi i t}}\right|^{2},
$$

and it follows that

$$
\begin{aligned}
& 4 Q_{N}(t) \sin ^{2} \pi t=\frac{1}{N} \sum_{n=1}^{N}\left|\left(1-e^{2 \pi i n t}\right)-\frac{1}{N} \sum_{m=1}^{N}\left(1-e^{2 \pi i m t}\right)\right|^{2} \\
& =\frac{1}{N} \sum_{n=1}^{N}\left|e^{2 \pi i n t}-\frac{1}{N} \sum_{m=1}^{N} e^{2 \pi i m t}\right|^{2}=\frac{1}{N} \sum_{n=1}^{N}\left|e^{2 \pi i n t}\right|^{2}-\left|\frac{1}{N} \sum_{n=1}^{N} e^{2 \pi i n t}\right|^{2}=1-\frac{\sin ^{2} \pi N t}{N^{2} \sin ^{2} \pi t} .
\end{aligned}
$$

We have thus obtained the formula

$$
Q_{N}(t)=\frac{1}{4 \sin ^{2} \pi t}\left(1-\frac{\sin ^{2} \pi N t}{N^{2} \sin ^{2} \pi t}\right) .
$$


The conclusion of (16) from this formula is immediate: in the case when the integral on the right hand side of (16) is finite one should merely apply to (17) the dominated convergence theorem, while in the case of divergence of the integral in (16) the result follows from Fatou's lemma.

4.2. We shall now consider the special case when $H=L^{2}(\mathbb{T})$ and the unitary operator $U$ is the irrational rotation,

$$
(U f)(x)=f(x+\alpha), \quad f \in L^{2}(\mathbb{T}),
$$

where $\alpha$ is a fixed irrational number. In this case the ergodic sums have the form

$$
S_{n}(x)=\sum_{k=0}^{n-1} f(x+k \alpha)
$$

Much attention has been paid to the case when these ergodic sums are bounded, that is, $\sup _{n}\left|S_{n}(x)\right|<\infty$ for some (or every) $x \in \mathbb{T}$. For more details we refer the reader to the papers $[\mathbf{4}, \mathbf{2 5}, \mathbf{2 7}, \mathbf{2 9}]$ and to the references therein.

Here we consider the bounded mean oscillation of the ergodic sums. In the next theorem we shall see that for a "good" function $f$, the BMO behavior of the ergodic sums implies that $f$ is a coboundary with respect to the rotation by $\alpha$.

Theorem 4.3. Let $\alpha$ be an irrational number, and $f$ be a Riemann integrable function on $\mathbb{T}$. Suppose that for some fixed $x_{0} \in \mathbb{T}$, the sequence $\left\{S_{n}\left(x_{0}\right)\right\}, n=1,2,3, \ldots$, belongs to BMO. Then there exists a function $g \in L^{2}(\mathbb{T})$ such that $f(x)=g(x)-g(x+\alpha)$ almost everywhere.

Recall the definition (2) of the space BMO given in Section 1. It is well-known that replacing the condition (2) with an appropriate $\ell^{p}$ version yields an equivalent definition of BMO. This is a consequence of a classical theorem of John and Nirenberg [8] (see also [5], Chapter VI §2) in a version adopted for sequences. In the next lemma we formulate this fact for $p=2$.

Lemma 4.4 ([8]). $\left\{c_{n}\right\} \in \mathrm{BMO}$ if and only if there is a constant $M$ such that

$$
\left(\frac{1}{m-n} \sum_{k=n}^{m-1}\left|c_{k}-\frac{c_{n}+\cdots+c_{m-1}}{m-n}\right|^{2}\right)^{1 / 2} \leqslant M
$$

for every $n<m$.

Proof of Theorem 4.3. Define

$$
v_{N}(x):=\left(\frac{1}{N} \sum_{n=1}^{N}\left|S_{n}(x)-\frac{S_{1}(x)+\cdots+S_{N}(x)}{N}\right|^{2}\right)^{1 / 2} \quad(x \in \mathbb{T}) .
$$

Using the obvious property $S_{n}(x+j \alpha)=S_{j+n}(x)-S_{j}(x)$ it follows that

$$
v_{N}(x+j \alpha)=\left(\frac{1}{N} \sum_{n=1}^{N}\left|S_{j+n}(x)-\frac{S_{j+1}(x)+\cdots+S_{j+N}(x)}{N}\right|^{2}\right)^{1 / 2} .
$$

The assumption that $\left\{S_{n}\left(x_{0}\right)\right\} \in$ BMO combined with Lemma 4.4 thus implies the existence of a constant $M$ such that

$$
v_{N}\left(x_{0}+j \alpha\right) \leqslant M, \quad j=0,1,2, \ldots .
$$


The function $v_{N}$ is therefore bounded by $M$ on a dense subset of $\mathbb{T}$. But $v_{N}$ is a Riemann integrable function, since so is $f$, hence it follows that

$$
\int_{\mathbb{T}} v_{N}(x)^{2} d x \leqslant M^{2}, \quad N=1,2, \ldots
$$

On the other hand, we have $\int v_{N}(x)^{2} d x=V_{N}$, where $V_{N}$ is the variance defined by (14). The variances are therefore bounded, so the proof is concluded by Theorem 4.1.

4.3. We can now prove Theorem 3. In fact, this theorem is a consequence of the following more general result.

Theorem 4.5. Let $\alpha$ be an irrational number, and $S \subset \mathbb{T}$ be a measurable set whose boundary has Lebesgue measure zero. Let $v(n, S)$ denote the number of integers $0 \leqslant k \leqslant$ $n-1$ such that $k \alpha \in S$. If the sequence $\{v(n, S)-n$ mes $S\}, n=1,2,3, \ldots$, belongs to BMO, then $\operatorname{mes} S \in \mathbb{Z} \alpha+\mathbb{Z}$.

The proof is a combination of the previous result with an argument due to Furstenberg, Keynes and Shapiro [4] and Petersen [27].

Proof of Theorem 4.5. The fact that the boundary of $S$ has Lebesgue measure zero means that the function $f(x)=\mathbb{1}_{S}(x)-$ mes $S$ is Riemann integrable. Let $S_{n}(x)$ be the ergodic sums of $f$ defined by (18). Then we have $\left\{S_{n}(0)\right\} \in$ BMO by assumption, and thus Theorem 4.3 implies the existence of a function $g \in L^{2}(\mathbb{T})$ such that $f(x)=g(x)-g(x+\alpha)$ (a.e.). Certainly we may also suppose that $g$ is real-valued.

Now consider the function $\tau(x)=\exp 2 \pi i g(x)$. We have

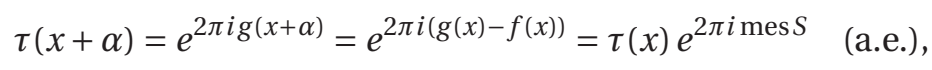

since the function $\mathbb{1}_{S}$ takes integer values. This means that $\tau$ is an eigenfunction of the irrational rotation by $\alpha$, with eigenvalue $\exp 2 \pi i$ mes $S$. However all the eigenvalues are known to be of the form $\exp 2 \pi i j \alpha, j \in \mathbb{Z}$, and therefore mes $S \in \mathbb{Z} \alpha+\mathbb{Z}$.

\section{Necessary condition for Riesz bases}

In this section we prove Theorem 2 . We will show that unless the diophantine condition $|I| \in \mathbb{Z} \alpha+\mathbb{Z}$ holds, the exponential system $E(\Lambda(\alpha, I))$ cannot be a Riesz basis in $L^{2}(S)$ for any multiband set $S$. The proof consists of three main ingredients: Meyer's duality principle, the results from Section 4 about discrepancy and BMO, and Pavlov's theorem describing the exponential Riesz bases in $L^{2}(I)$ where $I$ is an interval.

5.1. We start with the formulation of Pavlov's theorem.

Let $f(x)$ be a locally integrable function on $\mathbb{R}$. If $J$ is a bounded interval on $\mathbb{R}$ then

$$
\frac{1}{|J|} \int_{J}\left|f(x)-f_{J}\right| d x
$$

is called the mean oscillation of $f$ over $J$, where $f_{J}=|J|^{-1} \int_{J} f(x) d x$ denotes the average of $f$ over $J$. If the mean oscillation is bounded uniformly over all intervals $J$, then we say that $f$ has bounded mean oscillation, $f \in \mathrm{BMO}(\mathbb{R})$.

For a discrete set $\Lambda \subset \mathbb{R}$ we denote by $n_{\Lambda}(x)$ the 'counting function' satisfying

$$
n_{\Lambda}(b)-n_{\Lambda}(a)=\#(\Lambda \cap[a, b)), \quad a<b,
$$

which is defined uniquely up to an additive constant. We will use the following version of Pavlov's theorem (see [7, p. 240]) formulated in terms of the counting function $n_{\Lambda}$. 
Theorem 5.1 (Hruščev, Nikol'skii, Pavlov [7]). Let $\Lambda=\left\{\lambda_{n}, n \in \mathbb{Z}\right\}$. The exponential system $E(\Lambda)$ is a Riesz basis in $L^{2}(0, a), a>0$, if and only if

(i) $\Lambda$ is separated, that is, $\inf _{n \neq m}\left|\lambda_{n}-\lambda_{m}\right|>0$;

(ii) $f(x)=n_{\Lambda}(x)-$ ax is a function in $\mathrm{BMO}(\mathbb{R})$;

(iii) There is $y>0$ such that the harmonic continuation $U_{f}(x+i y)$ of $f$ into the upper half plane admits the following representation:

$$
U_{f}(x+i y)=c+\widetilde{u}(x)+v(x), \quad x \in \mathbb{R},
$$

where $c$ is a constant, $u, v$ are bounded measurable functions, $\|v\|_{\infty}<\frac{1}{4}$, and $\widetilde{u}$ is the Hilbert transform of $u$.

In the proof below we shall exploit only part (ii) of Theorem 5.1. We therefore do not discuss part (iii) in more detail. See [7] for a complete exposition.

5.2. We can finally prove the necessity of the condition $|I| \in \mathbb{Z} \alpha+\mathbb{Z}$.

Proof of Theorem 2. Suppose that $E(\Lambda(\alpha, I))$ is a Riesz basis in $L^{2}(S)$, for some multiband set $S \subset \mathbb{T}$. We may suppose that $S$ is semi-closed. Corollary 2.2, with $U=S$ and $V=I$, implies that $E(\Lambda(\alpha, S))$ is a Riesz basis in $L^{2}(-I)$. Using part (ii) of Theorem 5.1 it follows that the function

$$
f(x)=n_{\Lambda(\alpha, S)}(x)-|I| x
$$

belongs to $\mathrm{BMO}(\mathbb{R})$.

Since $\Lambda(\alpha, S)$ is a subset of $\mathbb{Z}$, the function $f$ is linear on each interval $(n-1, n]$ and has slope $-|I|$ there. It is therefore possible to write $f$ as the sum of two functions, $f=g+h$, where $g(x)$ is a piecewise constant function which is equal to $f(n)$ on each interval $(n-1, n]$, and $h(x)$ is a 1-periodic function which is linear with slope $-|I|$ on each such an interval. Thus,

$$
\frac{1}{m-n} \sum_{k=n+1}^{m}\left|f(k)-\frac{f(n+1)+\cdots+f(m)}{m-n}\right|
$$

is the mean oscillation of $g$ over the interval $(n, m]$. But since $g$ differs from $f$ by a bounded function, also $g \in \mathrm{BMO}(\mathbb{R})$. This implies that (20) is bounded uniformly with respect to $n$ and $m$, that is, the sequence $\{f(n)\}, n=1,2,3, \ldots$, belongs to BMO.

Recall that Landau's inequalities imply that mes $S=|I|$. By adding an appropriate constant to the counting function $n_{\Lambda(\alpha, S)}$ we may assume that $n_{\Lambda(\alpha, S)}(0)=0$. This means that

$$
f(n)=v(n, S)-n \operatorname{mes} S, \quad n=1,2,3, \ldots,
$$

where $v(n, S)$ denotes the number of integers $0 \leqslant k \leqslant n-1$ such that $k \alpha \in S$. Since we know that $\{f(n)\} \in$ BMO, it follows from Theorem 4.5 that mes $S \in \mathbb{Z} \alpha+\mathbb{Z}$. We conclude that $|I| \in \mathbb{Z} \alpha+\mathbb{Z}$ and so the theorem is proved.

\section{Remarks}

6.1. If $S$ is a single interval, then the complete description of the exponential Riesz bases $E(\Lambda)$ in $L^{2}(S)$ is given by Pavlov's theorem. Much less is known, however, in the case when $S$ is the union of two intervals or more. In fact, it is unknown in general whether a Riesz basis $E(\Lambda)$ (where $\Lambda \subset \mathbb{R}$ ) in $L^{2}(S)$ exists at all. This existence has been established in the following special cases: 
(i) $S$ is a finite union of disjoint intervals with commensurable lengths $[\mathbf{2}, \mathbf{1 6}]$.

(ii) $S$ is the union of two general intervals [30] (in this paper there is also a partial result for the union of more than two intervals).

Theorem 3.1 exhibits a new family of examples of multiband sets $S$ with a Riesz basis of exponentials, namely, every set $S$ with the structure (9). Moreover, in these examples the Riesz basis constructed consists of exponentials with integer frequencies.

A result of similar type in the non-periodic setting is given in [15].

We also mention the paper [17] where the authors construct, for any finite union of intervals, a Riesz basis of exponentials with complex frequencies lying in a horizontal strip along the real axis (see also [16]).

6.2. We mention several questions which are left open.

(a) For which multiband sets $S \subset \mathbb{T}$, mes $S=|I|$, is the exponential system $E(\Lambda(\alpha, I))$ a Riesz basis in $L^{2}(S)$ ?

In the case when $|I| \in \mathbb{Z} \alpha+\mathbb{Z}$ it was proved that condition (9) is sufficient for $S$ (Theorem 3.1), but we have not classified completely all such multiband sets $S$. It was also proved that no such sets exist if $|I| \notin \mathbb{Z} \alpha+\mathbb{Z}$ (Theorem 2).

(b) For which multiband sets $S \subset \mathbb{T}$ does the discrepancy $D(n, S)=v(n, S)-n$ mes $S$ (with respect to the irrational number $\alpha$ ) have bounded mean oscillation?

Theorem 4.5 says that mes $S \in \mathbb{Z} \alpha+\mathbb{Z}$ is a necessary condition for that.

As for the boundedness of the discrepancy, a necessary and sufficient condition is known to be the condition (9). This result was proved by Oren in [25] (see also [29]). The following question therefore seems natural:

(c) If $S$ is a multiband set such that $\{D(n, S)\} \in \mathrm{BMO}$, must it have the structure (9)?

If the answer to (c) is affirmative, then this would extend the dichotomy given by Theorem 3 for single intervals: either the discrepancy is bounded, or it does not even have bounded mean oscillation. It would also settle questions (a) and (b).

6.3. In connection with 'universal' sampling and interpolation, the result of Olevskii and Ulanovskii mentioned above (Theorem A of Section 1) shows, in particular, that given any $d>0$ one can find a system of exponentials with real frequencies, which is a Riesz basis in $L^{2}(S)$ for a "dense" family of multiband sets $S \subset \mathbb{R}$, mes $S=d$.

Here we have discussed the problem in the periodic setting, where an additional restriction is that the exponentials should have integer frequencies. Theorem $1 \mathrm{implies}$ that given any irrational number $d \in(0,1)$ one can find a system of exponentials with integer frequencies, which is a Riesz basis in $L^{2}(S)$ for a "dense" family of multiband sets $S \subset \mathbb{T}$, mes $S=d$.

It would be interesting to know what can be said if $d$ is a rational number. Compare also with the results about universal completeness of exponentials given in [24].

\section{References}

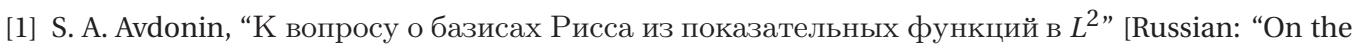

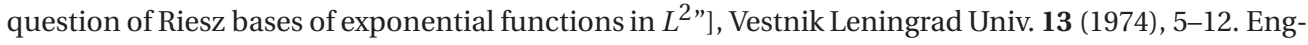
lish translation in Vestnik Leningrad Univ. Math. 7 (1979), 203-211. 
[2] L. Bezuglaya, V. Katsnelson, “The sampling theorem for functions with limited multi-band spectrum", Z. Anal. Anwendungen 12 (1993), 511-534.

[3] P. Erdös, "Problems and results on diophantine approximations", Compositio Math. 16 (1964), 52-65.

[4] H. Furstenberg, H. Keynes, L. Shapiro, "Prime flows in topological dynamics", Israel J. Math. 14 (1973), $26-38$.

[5] J. B. Garnett, "Bounded analytic functions", Academic Press, New York, 1981.

[6] E. Hecke, "Über analytische Funktionen und die Verteilung von Zahlen mod. eins" [German: "On analytic functions and the distribution of numbers mod one”], Abh. Math. Sem. Univ. Hamburg 1 (1922), 54-76.

[7] S. V. Hruščev, N. K. Nikol'skii, B. S. Pavlov, "Unconditional bases of exponentials and of reproducing kernels", Complex analysis and spectral theory (Leningrad, 1979/1980), pp. 214-335, Lecture Notes in Math. 864, Springer, Berlin, 1981.

[8] F. John, L. Nirenberg, “On functions of bounded mean oscillation”, Comm. Pure Appl. Math. 14 (1961), 415-426.

[9] Y. Katznelson, “An introduction to harmonic analysis", 2nd ed., Dover, New York, 1976.

[10] H. Kesten, “Uniform distribution mod 1", Ann. of Math. (2) 71 (1960), 445-471.

[11] H. Kesten, "Uniform distribution mod 1. II", Acta Arith. 7 (1961/1962), 355-380.

[12] H. Kesten, "On a conjecture of Erdös and Szüsz related to uniform distribution mod 1", Acta Arith. 12 (1966), 193-212.

[13] L. Kuipers, H. Niederreiter, “Uniform distribution of sequences”, Wiley, New York, 1974.

[14] H. J. Landau, "Necessary density conditions for sampling and interpolation of certain entire functions", Acta Math. 117 (1967) 37-52.

[15] N. Lev, "Riesz bases of exponentials on multiband spectra", preprint, arXiv:1101.3894.

[16] Yu. Lyubarskii, K. Seip, "Sampling and interpolating sequences for multiband-limited functions and exponential bases on disconnected sets", J. Fourier Anal. Appl. 3 (1997), 597-615.

[17] Yu. Lyubarskii, I. Spitkovsky, "Sampling and interpolation for a lacunary spectrum", Proc. Roy. Soc. Edinburgh Sect. A 126 (1996), 77-87.

[18] B. Matei, Y. Meyer, “Quasicrystals are sets of stable sampling”, C. R. Acad. Sci. Paris, Ser. I 346 (2008), $1235-1238$.

[19] B. Matei, Y. Meyer, “A variant of compressed sensing”, Rev. Mat. Iberoam. 25 (2009), 669-692.

[20] B. Matei, Y. Meyer, "Simple quasicrystals are sets of stable sampling”, Complex Var. Elliptic Equ. 55 (2010), 947-964.

[21] Y. Meyer, "Nombres de Pisot, nombres de Salem et analyse harmonique" [French: "Pisot numbers, Salem numbers and harmonic analysis"], Lecture Notes in Mathematics 117, Springer-Verlag, BerlinNew York, 1970.

[22] Y. Meyer, "Algebraic numbers and harmonic analysis", North-Holland, Amsterdam, 1972.

[23] A. Olevskii, A. Ulanovskii, "Universal sampling of band-limited signals", C. R. Acad. Sci. Paris, Ser. I 342 (2006), 927-931.

[24] A. Olevskii, A. Ulanovskii, "Universal sampling and interpolation of band-limited signals", Geom. Funct. Anal. 18 (2008), 1029-1052.

[25] I. Oren, "Admissible functions with multiple discontinuities", Israel J. Math. 42 (1982), 353-360.

[26] B. S. Pavlov, “Базисность системы экспонент и условие Макенхаупта” [Russian: “The basis property of a system of exponentials and the condition of Muckenhoupt"], Dokl. Akad. Nauk SSSR 247 (1979), 37-40. English translation in Soviet Math. Dokl. 20 (1979), 655-659.

[27] K. Petersen, "On a series of cosecants related to a problem in ergodic theory", Compositio Math. 26 (1973), 313-317.

[28] E. A. Robinson, "Sums of stationary random variables", Proc. Amer. Math. Soc. 11 (1960), 77-79.

[29] J. Schoissengeier, "Regularity of distribution of ( $n \alpha)$-sequences", Acta Arith. 133 (2008), 127-157.

[30] K. Seip, "A simple construction of exponential bases in $L^{2}$ of the union of several intervals", Proc. Edinburgh Math. Soc. 38 (1995), 171-177.

[31] R. M. Young, “An introduction to nonharmonic Fourier series", Academic Press, New York, 1980.

Department of Mathematics, Weizmann Institute of Science, Rehovot 76100, Israel.

E-mail address: gady.kozma@weizmann.ac.il

Department of Mathematics, Weizmann Institute of Science, Rehovot 76100, IsRael.

E-mail address: nir.lev@weizmann. ac.il 